\title{
THE STRUCTURE OF HOMOMORPHISMS FROM BANACH ALGEBRAS OF DIFFERENTIABLE FUNCTIONS INTO FINITE DIMENSIONAL BANACH ALGEBRAS
}

\author{
VIET NGO \\ Department of Mathematics and Computer Science \\ California State University, Long Beach \\ Long Beach, California 90840
}

(Received June 1, 1988)

\begin{abstract}
We show that the structure of continuous and discontinuous homomorphisms from the Banach algebra $C^{n}[0,1]$ of $\mathrm{n}$ times continuously differentiable functions on the unit interval $[0,1]$ into finite dimensional Banach algebras is completely determined by higher point derivations.
\end{abstract}

KEY WORDS AND PHRASES. Banach algebras, homomorphisms, local algebras, singularity set, higher point derivations.

1980 MATHEMATICS SUBJECT CLASSIFICATION CODE. $46 \mathrm{~J} 10$

\section{Introduction.}

It is well known that the Banach algebra $C^{n}[0,1]$ is generated by $x(t)=t, 0 \leq t \leq 1$. Thus a continuous homomorphism $\nu$ of $C^{n}[0,1]$ into a Banach algebra 9 is completely determined by $\nu(z)$. We are mainly interested in the structure of discontinuous homomorphisms $\nu$ from $C^{n}[0,1]$ into finite dimensional Banach algebras. In 1980 Bade, Curtis and Laursen [1] showed that these homomorphisms have a striking degree of continuity: the restriction of $\nu$ to $C^{2 n}[0,1]$ is continuous with respect to the $C^{2 n}$-norm. So, if we can obtain an explicit structure of continuous homomorphism $\nu$ from $C^{n}[0,1]$ into finite dimensional Banach algebras we may understand the behavior of discontinuous ones; that will be our approach to this problem.

\section{Preliminaries.}

Let $C^{n}[0,1]$ denote the algebra of all complex valued functions on $[0,1]$ which have $\mathrm{n}$ continuous derivatives. It is well known that $C^{n}[0,1]$ is a Banach algebra under the norm

$$
\|f\|=\max _{t \in[0,1]} \sum_{k=0}^{n} \frac{\left|f^{(k)}(t)\right|}{k !}
$$

whose structure space is $[0,1]$. We will need a characterization of the square of the closed primary ideals with finite codimension in $C^{n}[0,1]$. We use the notation

$$
\mathrm{M}_{n, k}\left(t_{0}\right)=\left\{f \in C^{n}[0,1] \mid f^{(j)}\left(t_{0}\right)=0 ; j=0,1, \ldots, k\right\} .
$$

These are precisely the closed ideals of finite codimension contained in the maximal ideal $\mathrm{M}_{n, k}\left(t_{0}\right)$ of functions vanishing at $t_{0}$. Writing $\mathrm{M}_{n, k}$ for $\mathrm{M}_{n, k}(0)$ and setting $\not(t)=t, 0 \leq t \leq 1$, we have: 
1.1 THEOREM. Let $n$ be a positive integer. Then

(i) $\mathrm{M}_{n, 0}^{2}=z \mathrm{M}_{n, 0}=\left\{f \mid f(0)=f^{\prime}(0)=0\right.$ and $f^{(n+1)}(0)$ exists $\}$,

(ii) $\mathrm{M}_{n, k}^{2}=z^{k+1} \mathrm{M}_{n, k}, 1 \leq k \leq n-1$,

(iii) $\mathrm{M}_{n, n}^{2}=z^{n} \mathrm{M}_{n, n}$.

Part (i) is from [2, Example 3]. Part (ii) is due to Dales and McClure [3,Theorem 3.1]. The proof of part (iii) can be found in [4].

The squares of the closed primary ideals $M_{n, k}\left(t_{0}\right)$ at other points $t_{0}$ in $[0,1]$ are given exactly by similar formulas, where $z$ is replaced by $z-t_{0}$. We also need the following concepts and facts in automatic continuity theory.

1.2 DEFINITION. If $\mathrm{T}: \ell \rightarrow \mathscr{B}$ is a linear map and $\ell, \mathscr{B}$ are Banach spaces, then the separating space of $\mathrm{T}, \boldsymbol{\varphi}(\mathrm{T})$ is defined by

$$
\mathscr{Y}(\mathrm{T})=\left\{y \in \mathscr{B} \mid \exists\left\{x_{n}\right\} \subset \curlywedge, x_{n} \Rightarrow 0 \text {, and } \mathrm{T}\left(x_{n}\right) \Rightarrow y\right\} .
$$

This space measures the discontinuity of $T$ because $\varphi(T)=\{0\}$ if and only if $T$ is discontinuous, by the closed graph theorem. More detailed discussion on $\varphi(T)$ can be found in [5].

1.3 DEFINITION. If $\boldsymbol{l}, \Phi$ are Banach algebras, and $\mathrm{T}: \boldsymbol{l} \rightarrow \mathscr{B}$ is a homomorphism with separating space $\varphi(T)$, then the continuity ideal of $T, g(T)$ is defined by

$$
g(T)=\{x \in \Lambda \mid T(x) \varphi(T)=(0)\} .
$$

Let $\Phi$ be a Banach algebra and $\nu: C^{n}[0,1] \rightarrow \Phi$ be a homomorphism. It is shown in $[6,7]$ that the continuity ideal $g(\nu)$ has finite hull and contains the ideal $J(F)$ of all functions vanishing in neighborhoods of $\mathrm{F}=\operatorname{hull}(\mathrm{I}(\nu)) . \mathrm{F}$ is called the singularity set $\mathrm{\nu} \nu$.

1.4 THEOREM. Let $n$ be a positive integer and $\nu: C^{n}[0,1] \rightarrow \Im$ be a discontinuous homomorphism with singularity set $F=\{0\}$. Consider the following statements:

(a) $Y(\nu)$ is finite dimensional,

(b) $\mathbf{g}(\nu)$ has finte codimension,

(c) $g(\nu)$ is closed and contains $M_{n, n-1}$,

(d) $\varphi(\nu)^{2}=\{0\}$,

(e) $z^{n} \in \mathrm{g}(\nu)$,

(f) $\nu$ is continuous on $\mathrm{M}_{n, n}^{2}=z^{n} \mathrm{M}_{n, n}$ for the graph norm $\|f\|\|=\| f\|+\| \frac{f}{z^{n}} \|$,

(g) $\nu$ is $C^{2 n}$-continuous (i.e. the restriction of $\nu$ to $C^{2 n}$ is continuous with respect to the $C^{2 n}$-norm).

We have the following implications:

For the proof see [1].

$$
(a) \Rightarrow(b) \Leftrightarrow(c) \Leftrightarrow \text { (d) } \Leftrightarrow(e) \Leftrightarrow \text { (f) } \Rightarrow(g)
$$

\section{Algebraic results.}

Let $\nu$ be a homomorphism of $C^{n}[0,1]$ into a finite dimensional Banach algebra 9 . We may assume that $\nu$ is onto by considering $\nu: C^{n}[0,1] \rightarrow \nu\left(C^{n}[0,1]\right)$. We shall reduce the study of $\nu$ to the case where the range space is a finite dimensional local algebra. 
Let 9 be the range of $\nu$. Since $9 B$ is a finite dimensional commutative algebra with a unit $e$, the Wedderburn principal theorem states that $\mathscr{B}=\boldsymbol{\Lambda} \Phi$, where 9 is the radical of $\mathscr{B}$. Now $\boldsymbol{l}$ is a semisimple commutative algebra with unit. By the Wedderburn structure theorem for finite dimensional algebras we can write

$$
\curlywedge=e_{1} \curlywedge \oplus \ldots \oplus e_{m} \curlywedge,
$$

where

$$
\begin{aligned}
e_{i} e_{j} & =0, & i \neq j, \\
e_{i}{ }^{2} & =e_{i}, & i=1,2, \ldots, m, \\
e & =e_{1}+\ldots+e_{m}, &
\end{aligned}
$$

and $e_{i} \mathcal{l}$ is a simple commutative algebra with unit $e_{i}$, so that $e_{i} \mathcal{C}$. Thus we can write

$$
\mathscr{B}=e_{1} \mathscr{B} \oplus \ldots \oplus e_{m} \mathscr{B},
$$

where each $e_{i}$ I is a local algebra which may be isomorphic to C. Moreover $\nu=e_{1} \nu+\ldots+e_{m} \nu$ and each $e_{i} \nu$ is a homomorphism of $C^{n}[0,1]$ onto $e_{i}$ 疋. If $e_{i}$ 疋 $\approx \mathrm{C}$ then $e_{i} \nu$ is just a multiplicative linear functional and is of the form $e_{i} \nu(f)=f\left(t_{0}\right) e_{i}$ for some $t_{0}$ in $[0,1]$. It remains to consider the case when $e_{i}$ क is a local algebra which is not isomorphic to $C$. Our next objective is to characterize the kernel of $\nu$.

2.1 LEMMA. Let $\nu$ be a homomorphism of $C^{n}[0,1]$ onto a finite dimensional algebra. If $t_{0} \in$ hull(ker $\nu)$ then

(i) $\left(z-t_{0}\right)^{m} \in$ ker $\nu$ for some positive integer $m$,

(ii) The ideal $\mathrm{J}\left(t_{0}\right)$ of all functions vanishing in neighborhoods of $t_{0}$ is contained in ker $\nu$.

PROOF: (i) Suppose that $\nu\left(z-t_{0}\right)$ is not nilpotent. Then $\nu\left(z-t_{0}\right)$ is invertible so that there exists $g$ in $C^{n}[0,1]$ such that $\nu\left(z-t_{0}\right) \nu(g)=\nu(1)$. Thus $\left(z-t_{0}\right) g=1+f$ for some $f$ in ker $\nu$. But $\left(z-t_{0}\right) g \in \mathrm{M}_{n, 0}\left(t_{0}\right)$ and $f \in \operatorname{ker} \nu \subset \mathrm{M}_{n, 0}\left(t_{0}\right)$ so that $1 \in \mathrm{M}_{n, 0}\left(t_{0}\right)$. This is a contradiction.

(ii) Let $f \in \mathrm{J}\left(t_{0}\right)$. Choose $g \in \mathrm{J}\left(t_{0}\right)$ such that $g$ is identically one on the support of $f$. We claim that $\nu(g)$ is nilpotent. Suppose not, then $\nu(g)$ is invertible and $e=\nu(h)$ for some $h \in \mathrm{J}\left(t_{0}\right)$. So $1=h+v$ for some $v \in \operatorname{ker} \nu$. This is a contradiction since $h \in \mathrm{J}\left(t_{0}\right) \subset \mathrm{M}_{n, 0}\left(t_{0}\right)$ and $v \in \mathrm{M}_{n, 0}\left(t_{0}\right)$. Thus $g^{m} \in \operatorname{ker} \nu$ for some $m$ and we have $f=f g^{m} \in \operatorname{ker} \nu$.

Immediately following this lemma we have:

2.2 COROLLARY. Let $\nu$ be a homomorphism of $C^{n}[0,1]$ onto a finite dimensional local algebra. The hull of $\operatorname{ker} \nu$ consists of exactly one point $t_{0}$ and therefore the singularity set $F=$ hull $(I(\nu)) \subseteq\left\{t_{0}\right\}$.

PROOF: Let $t_{0}$ and $t_{1}$ be in hull(ker $\left.\nu\right)$. By 2.1 there exist positive integers $m_{0}$ and $m_{1}$ such that $\nu\left(z-t_{0}\right)^{m_{0}}=0$ and $\nu\left(z-t_{1}\right)^{m_{1}}=0$. Then $\nu\left[\left(z-t_{0}\right)-\left(z-t_{1}\right)\right]^{m_{0}+m_{1}}=0$

so that $t_{1}-t_{0}=0$. Thus hull $(\operatorname{ker} \nu)=t_{0}$ for some $t_{0}$ in $[0,1]$. Since $\operatorname{ker} \nu \subseteq g(\nu)$ it follows that hull $(g(\nu)) \subseteq\left\{t_{0}\right\}$.

Without loss of generality we shall take hull $(\operatorname{ker} \nu)$ to be $\{0\}$. With this assumption we now describe $\nu$ for the case when it is continuous. 
2.3 THEOREM. Let $\nu$ be a continuous homomorphism of $C^{n}[0,1]$ onto a finite dimensional local algebra with hull $(\operatorname{ker} \nu)=\{0\}$. There exists a positive integer $k \leq n+1$ such that

where $\delta_{i}(f)=\frac{f^{(i)}(0)}{i !}$

$$
\nu(f)=\sum_{i=1}^{k-1} \delta_{i}(f) \nu(z)^{i}
$$

PROOF: Since $\nu$ is continuous, ker $\nu$ is a closed primary ideal of finite codimension. Thus ker $\nu=\mathrm{M}_{n, k-1}$ for some $k \leq n+1$. Let $f \in C^{n}[0,1]$, we write $f=\sum_{i=1}^{k-1} \delta_{i}(f) z^{i}+R f$, where $R f \in$ $\mathrm{M}_{n, k-1}=\operatorname{ker} \nu$. Then

$$
\nu(f)=\sum_{i=0}^{k-1} \delta_{i}(f) \nu(z)^{i}
$$

The sequence of linear functionals $\delta_{1}, \ldots, \delta_{k-1}$ is called a continuous higher point derivation of order $\mathrm{k}-1$ on $C^{n}[0,1]$ at $\delta_{0}$. We refer to [3] for a complete description of the order and continuity properties of higher point derivations on $C^{n}[0,1]$.

\section{The structure of discontinuous homomorphisms of $C^{n}[0,1]$ onto finite dimensional local algebras.}

We now turn our attention to discontinuous homomorphisms $\nu$ of $C^{n}[0,1]$ onto a finite dimensional local algebra $\mathscr{B}$ with hull $(\operatorname{ker} \nu)=\{0\}$. First we characterize $\operatorname{ker} \nu$.

3.1 LEMMA. $\overline{\operatorname{ker} \nu}=\nu^{-1}(\varphi(\nu))$.

PROOF: Let $f \in \overline{\operatorname{ker} \nu}$, there exists $\left\{f_{m}\right\} \subset$ ker $\nu$ with $f_{m} \Rightarrow f$. Then $f-f_{m} \Rightarrow 0$ and $\nu\left(f-f_{m}\right)$ $\Rightarrow \nu(f)$ so that $\nu(f) \in \varphi(\nu)$. Hence $\overline{\operatorname{ker} \nu} \subseteq \nu^{-1}(\varphi(\nu))$.

Now let $f \in \nu^{-1}(\varphi(\nu))$. By definition of $\varphi(\nu)$, there exists $\left\{f_{k}\right\} \subset C^{n}[0,1]$ with $f_{k} \Rightarrow 0$ and $\nu\left(f_{k}\right) \Rightarrow$ $\nu(f)$. Since $\overline{\operatorname{ker} \nu}$ has finite codimension in $C^{n}[0,1]$, there exists a subspace $\mathrm{V}$ with $\operatorname{dim} \mathrm{V}<\infty$ such that $C^{n}[0,1]=\overline{\operatorname{ker} \nu} \oplus \mathrm{V}$. So we can write $f_{k}=g_{k}+v_{k}$ where $g_{k} \in \overline{\operatorname{ker} \nu}$ and $v_{k} \in \mathrm{V}$. But $f_{k} \Rightarrow 0$ so that $g_{k} \Rightarrow 0$ and $v_{k} \Rightarrow 0$. Since $\operatorname{dim} V<\infty, \nu\left(v_{k}\right) \Rightarrow 0$ so that $\nu\left(g_{k}\right)=\nu\left(f_{k}\right)-\nu\left(v_{k}\right) \Rightarrow \nu(f)$. Again we can write $\overline{\operatorname{ker} \nu}=\operatorname{ker} \nu \oplus \mathrm{W}$, where $\operatorname{dim} \mathrm{W}<\infty$, so $g_{k}=h_{k}+w_{k}$ where $h_{k} \in \operatorname{ker} \nu$ and $w_{k} \in \mathrm{W}$. Then $\nu\left(g_{k}\right)=\nu\left(w_{k}\right) \Rightarrow \nu(f)$ so that $\nu(f) \in \nu(\mathrm{W})$. Thus $f \in \mathrm{W}+\operatorname{ker} \nu=\overline{\operatorname{ker} \nu}$ and we conclude that $\nu^{-1}(\varphi(\nu)) \subseteq \overline{\operatorname{ker} \nu}$.

3.2 LEMMA. Let $k$ be the integer for which $\overline{\operatorname{ker} \nu}=\mathrm{M}_{n, k-1}, k \leq n+1$. Then $\mathrm{M}_{n, k-1}^{2} \subseteq$ $\operatorname{ker} \nu$ and

$$
\mathscr{B}=\operatorname{span}\left\{e, \nu(z), \ldots, \nu(z)^{k-1}\right\} \oplus \varphi(\nu) .
$$

PROOF: The first statement is clear since $\overline{\operatorname{ker} \nu}$ is a closed primary ideal of finite codimension. By $3.1 \nu\left(\mathrm{M}_{n, k-1}\right)=\varphi(\nu)$. Let $f, g \in \mathrm{M}_{n, k-1}$, then $\nu(f g)=\nu(f) \nu(g) \in \varphi(\nu)^{2}=\{0\}$ by 1.4 . Since

$$
C^{n}[0,1]=\operatorname{span}\left\{1, z, \ldots, z^{k-1}\right\} \oplus \overline{\operatorname{ker} \nu}
$$

we have

$$
\mathscr{B}=\nu\left(C^{n}[0,1]\right)=\operatorname{span}\left\{e, \nu(z), \ldots, \nu(z)^{k-1}\right\}+\varphi(\nu)
$$

by 3.1 . To see that the sum is direct let

$$
b=a_{0} e+a_{1} \nu(z)+\ldots+a_{k-1} \nu(z)^{k-1} \in Y(\nu)
$$

and suppose $b \neq 0$. Let $j$ be the smallest integer such that $a_{j} \neq 0$, then 


$$
\nu(z)^{j}\left\{\sum_{i=j}^{k-1} a_{i} \nu(z)^{i-j}\right\} \in \varphi(\nu)
$$

But $\sum_{i=j}^{k-1} a_{i} \nu(z)^{i-j}$ is invertible since $a_{j} \neq 0$, so $\nu(z)^{j} \in \mathcal{Y}(\nu)$. By $3.1 z^{j} \in \overline{\operatorname{ker} \nu}=\mathrm{M}_{n, k-1}$. This is a contradiction since $j \leq k-1$.

We are now in position to describe discontinuous homomorphisms.

3.3 THEOREM. Let $\nu$ be a discontinuous homomorphism of $C^{n}[0,1]$ onto a finite dimensional local algebra 9 with hull $(\operatorname{ker} \nu)=\{0\}$ and $\overline{\operatorname{ker} \nu}=\mathrm{M}_{n, k-1}$. There exist $b_{1}, \ldots, b_{m}$ in $\varphi(\nu)$ and discontinuous linear functionals $\gamma_{1}, \ldots, \gamma_{m}$ on $C^{n}[0,1]$ which vanish on polynomials and on the principal ideal $z^{k} C^{n}[0,1]$ such that

$$
\nu(f)=\sum_{l=0}^{k+k_{1}} d_{l}(f) \nu(z)^{l}+\sum_{l=0}^{i_{1}} \gamma_{1}\left(z^{i_{1}-l} f\right) \nu(z)^{l} b_{1}+\ldots+\sum_{l=0}^{i_{m}} \gamma_{m}\left(z^{i_{m}-l} f\right) \nu(z)^{l} b_{m},
$$

$0 \leq k_{1}, i_{1}, \ldots, i_{m} \leq k-1$,

where $d_{1}, \ldots, d_{k}+k_{1}$ is a higher point derivation at 0 and the linear functionals $\theta_{j}$ defined by $\theta_{j}(f)$ $=\gamma_{j}\left(z^{i_{j}} f\right), j=1, \ldots, m$, are discontinuous point derivations at 0 .

PROOF: Since $z^{k} \in M_{n, k-1}=\overline{\operatorname{ker} \nu}=\nu^{-1}(\varphi(\nu))$ and $\varphi(\nu)^{2}=\{0\}$, the multiplication operator $\nu(z): \varphi(\nu) \rightarrow \varphi(\nu)$ is nilpotent of index less than or equal to $k$. So we may choose a basis $B$ for $\varphi(\nu)$ of the form

$$
B=\left\{\nu(z)^{k}, \ldots, \nu(z)^{k+k_{1}}, b_{1}, \nu(z) b_{1}, \ldots, \nu(z)^{i_{1}} b_{1}, \ldots, b_{m}, \nu(z) b_{m}, \ldots, \nu(z)^{i_{m}} b_{m}\right\}
$$

where $0 \leq k_{1}, i_{1}, \ldots, i_{m} \leq k-1$. Let $f \in C^{n}[0,1]$. Consider the Taylor expansion

$$
f=\sum_{i=0}^{k-1} \delta_{i}(f) z^{i}+R f
$$

where $R f \in \mathrm{M}_{n, k-1}=\overline{\operatorname{ker} \nu}$. Since $\nu(R f) \in \mathcal{Y}(\nu)$ we can write

$$
\begin{gathered}
\nu(f)=\sum_{l=0}^{k-1} \delta_{l}(f) \nu(z)^{l}+\sum_{l=k}^{k+k_{1}} d_{l}(f) \nu(z)^{l}+\sum_{l=0}^{i_{l}} \gamma_{1, l+1}(f) \nu(z)^{l} b_{1}+\ldots+ \\
\quad+\sum_{l=0}^{i_{m}} \gamma_{m, l+1}(f) \nu(z)^{l} b_{m}
\end{gathered}
$$

We make the following observations:

(i) The coefficient functionals $d_{k}, \ldots, d_{k+k_{1}}, \gamma_{1,1}, \ldots, \gamma_{1, i_{1}+1}, \ldots, \gamma_{m, 1}, \ldots, \gamma_{m, i_{m+1}}$ are discontinuous. To see this, consider $\gamma_{1,1}$. Since $b_{1} \in \mathcal{Y}(\nu)$, there exist $f_{j} \Rightarrow 0$ in $C^{n}[0,1]$ with $\nu\left(f_{j}\right)$ $\Rightarrow b_{1}$. We have

$$
\begin{aligned}
& \nu\left(f_{j}\right)-b_{1}=\sum_{l=0}^{k-1} \delta_{l}\left(f_{j}\right) \nu(z)^{l}+\sum_{l=k}^{k+k_{1}} d_{l}\left(f_{j}\right) \nu(z)^{l}+\left(\gamma_{1,1}\left(f_{j}\right)-1\right) b_{1}+ \\
& \quad+\sum_{l=1}^{i_{1}} \gamma_{1, l+1}\left(f_{j}\right) \nu(z)^{l} b_{1}+\sum_{l=0}^{i_{2}} \gamma_{2, l+1}\left(f_{j}\right) \nu(z)^{l} b_{2}+\ldots+\sum_{l=0}^{i_{m}} \gamma_{m, l+1}\left(f_{j}\right) \nu(z)^{l} b_{m}
\end{aligned}
$$


Since $\nu\left(f_{j}\right)-b_{1} \Rightarrow 0$ we must have all coefficients tending to zero as $j \rightarrow \infty$, in particular $\lim _{j \rightarrow \infty} \gamma_{1,1}\left(f_{j}\right)=1$, which implies that $\gamma_{1,1}$ is discontinuous. The same argument works for the other coefficient functionals $d_{k}, \ldots, d_{k+k_{1}}, \gamma_{1,2}, \ldots, \gamma_{1, i_{1}+1}, \ldots, \gamma_{m}, 1, \ldots, \gamma_{m}, i_{m+1}$.

(ii) Since $z^{k} \mathrm{M}_{n, k-1}=\mathrm{M}_{n, k-1}^{2} \subseteq \operatorname{ker} \nu$ (by 3.2) all the above functionals vanish on $z^{k} \mathrm{M}_{n, k-1}$. For a notational purpose we set $d_{l}=\delta_{l}$ for $l=0,1, \ldots, k-1$. Let $f, g \in C^{n}[0,1]$. Using the fact that $\nu(z)^{k+k_{1}+1}=0$ and $y(\nu)^{2}=\{0\}$ (by 1.4), we have

$$
\begin{aligned}
\nu(f) \nu(g)=\sum_{l=0}^{k+k_{1}}\left[\sum_{j=0}^{l} d_{j}(f) d_{l}-j(g)\right] \nu(z)^{l} \\
+\sum_{l=0}^{i_{1}}\left[\sum_{j=0}^{l} d_{j}(f) \gamma_{1, l+1-j}(g)+\gamma_{1, l+1-j}(f) d_{j}(g)\right] \nu(z)^{l} b_{1}+\ldots . \\
\quad+\sum_{l=0}^{i_{m}}\left[\sum_{j=0}^{l} d_{j}(f) \gamma_{m, l+1-j}(g)+\gamma_{m, l+1-j}(f) d_{j}(g)\right] \nu(z)^{l} b_{m}
\end{aligned}
$$

Since $\nu(f) \nu(g)=\nu(f g)$ we have

(iii) $d_{l}(f g)=\sum_{j=0}^{l} d_{j}(f) d_{l-j}(g)$ for $0 \leq l \leq k+k_{1}+1$, so $d_{1}, \ldots, d_{k+k_{1}}$ is a higher point derivation at 0 .

(iv) For $j=1, \ldots, m$ and $l=1, \ldots, i_{j}$ we have $\gamma_{j, l+1}\left(z^{i}\right)=0, i=0,1,2, \ldots$. Because $\gamma_{j}, l+1\left(z^{i}\right)=$ 0 , for $i=0,1, \ldots, k+k_{1}$ since $d_{j}\left(z^{i}\right)=0$ if $i \neq j, d_{j}\left(z^{i}\right)=1$ if $i=j$, and $\operatorname{span}\left\{e, \nu(z), \ldots, \nu(z)^{k-1}\right\} \oplus$ $\operatorname{span} B=9 . \gamma_{j, 1+1}\left(z^{i}\right)=0$, for $i \geq k+k_{1}+1$ since $\nu(z)^{k+k_{1}+1}=0$.

Combining (ii) and (iv) we see that all the $\gamma_{j, 1+1}$ vanish on $z^{k} C^{n}[0,1]$.

(v) For $j=1, \ldots, m$ and $l=1, \ldots, i_{j}$ we have

so that

$$
\gamma_{j, l+1}(f g)=\sum_{s=0}^{l} \delta_{s}(f) \gamma_{j, l+1}-.(g)+\gamma_{j, l+1}-.(f) \delta_{s}(g)
$$

$$
\gamma_{j, i_{j}+1}\left(z^{i_{j}-1} f\right)=\gamma_{j, l+1}(f), f \in C^{n}[0,1], j=1, \ldots, m \text {. }
$$

We take $\gamma_{j}=\gamma_{j, i_{j}+1}, j=1, \ldots, m$. Letting $l=0$ in (v), we note that the linear functionals $\theta_{j}$, $(j=1, \ldots, m)$, defined by $\theta_{j}(f)=\gamma_{j}, 1(f)=\gamma_{j}\left(z^{i_{j}} f\right)$ are discontinuous point derivations at 0 .

\section{REFERENCES}

1. W.G. Bade and P.C. Curtis,Jr., and K.B. Laursen, Divisible subspaces and problems of automatic continuity, Studia Mathematica, T. LXVIII (1980), 159-186.

2. W.G. Bade and P.C. Curtis, Jr. The continuity of derivations of Banach algebras, J. Functional Analysis 16 (1974), 372-387.

3. H.G. Dales and J.P. McClure, Higher point derivations on commutative Banach algabras, I, J. Functional Analysis 26 (1977), 166-189.

4. W.G. Bade and P.C. Curtis, Jr. The structure of module derivations of Banach algebras of differentiable functions, J. Functional Analysis 28 (1978), 226-247.

5. A.M. Sinclair, Automatic continuity of linear operators, London Math. Soc. Lecture note series 21, C.U.P., Cambridge 1976.

6. W.G. Bade and P.C. Curtis, Jr. Homomorphisms of commutative Banach algebras, Amer. J. Math 82 (1960), 589-608.

7. W.G. Bade and P.C. Curtis, Jr., and K.B. Laursen, Automatic continuity in algebras of differentiable functions, Math. Scand. $\underline{40}$ (1977), 249-270. 


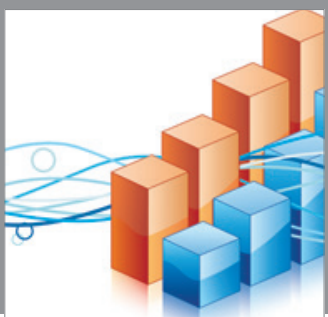

Advances in

Operations Research

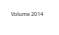

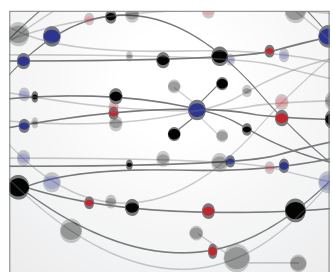

\section{The Scientific} World Journal
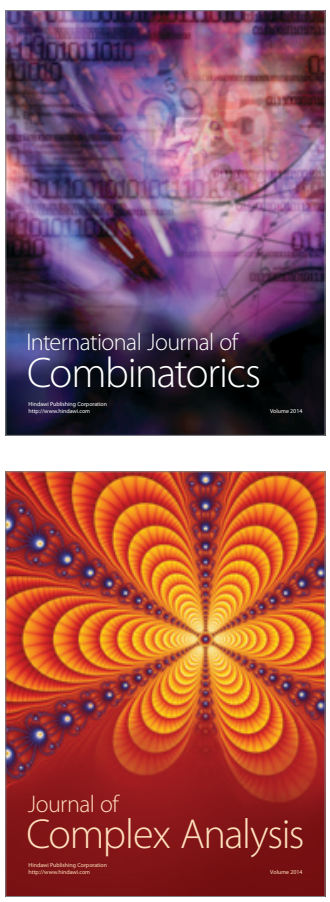

International Journal of

Mathematics and

Mathematical

Sciences
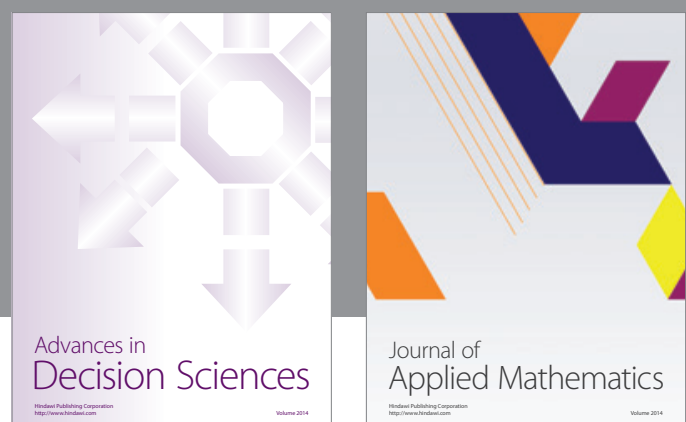

Journal of

Applied Mathematics
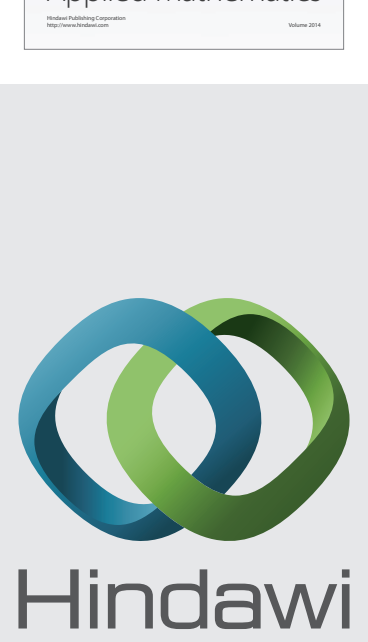

Submit your manuscripts at http://www.hindawi.com


Mathematical Problems in Engineering
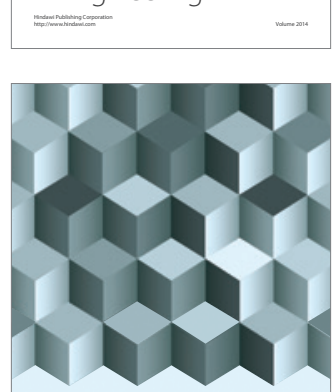

Journal of

Function Spaces
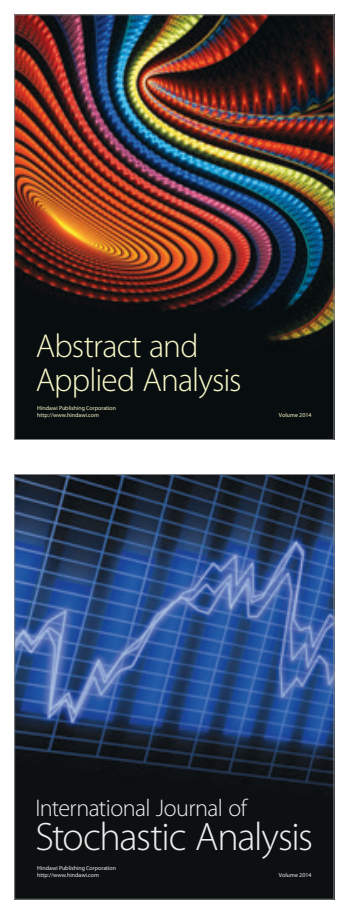

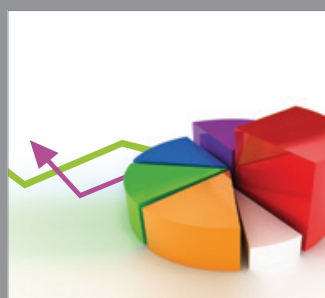

ournal of

Probability and Statistics

Promensencen
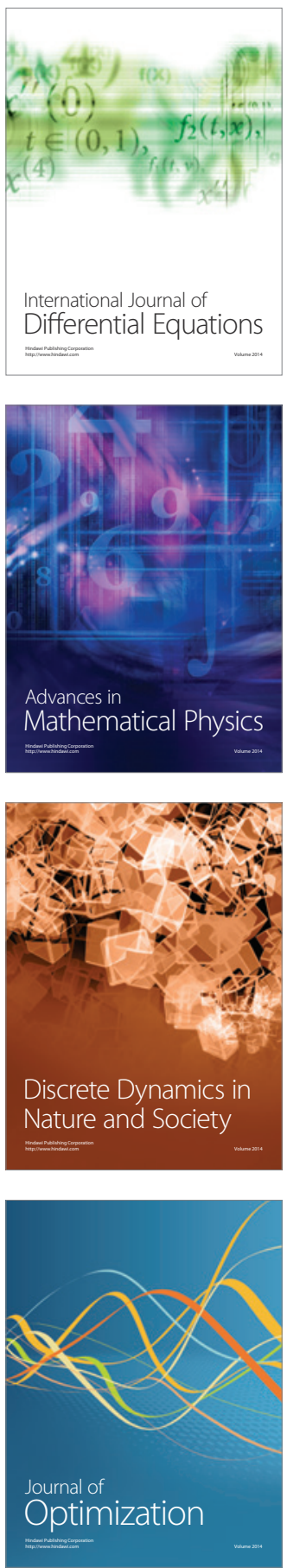